\title{
BATIMETRÍA Y ANÁLISIS MORFOMÉTRICO DEL LAGO DE ARREO (N. ESPAÑA)
}

\author{
E. Rico, A. Chicote, M.E. González y C. Montes \\ Departamento Interuniversitario de Ecología. Facultad de Ciencias. Universidad Autónoma de Madrid. 28049 Madrid. España.
}

Palabras clave: Batimetría, morfometría, lagos de disolución, País Vasco, España.

Key words: bathymetry, lake morphometry, solution lake, lake in evaporitic substrata. Basque Country. Spain.

\begin{abstract}
BATHYMETRY AND MORPHOMETRICAL ANALYSIS OF THE LAKE OF ARREO (N. SPAIN)

A bathymetric map of the solution lake of Arreo (N. Spain) based on echo-sounding is presented. Also, a morphometrical study based on this bathymetry is made. Sorne ecological consequences are denoted.
\end{abstract}

\section{INTRODUCCIÓN}

El lago de Arreo está situado en el País Vasco, a 655 m. (UTM 30TWN0036), y pertenece a la localidad alavesa de Caicedo de Yuso. Se localiza en la cuenca medio-alta del río Ebro, en el borde sur del Diapiro dc Salinas de Añana, principal chimenea salina dentro de las numerosas que se presentan en la denominada Fosa Cantábrica (RÍOS, 1963). Su cubeta y cuenca se asientan sobre materiales triásicos del Keuper formados por arcillas abigarradas y yesos con ofitas intercaladas.

Posee una morfogénesis kárstica en yesos. Son numerosas las formaciones palustres originadas por procesos de disolución en los materiales diapíricos de la Fosa Cantábrica, como la laguna de Bárcena y las de Las Lamas (Burgos) o, en el mismo País Vasco, los complejos de humedales de los diapiros de Maestu y Altube. En este contexto, Arreo es el cuerpo de agua de estas características más importante, con profundidad suficiente para considerarse limnológicamente como un lago, es decir, un ecosistema acuático cuya organización y dinámica se centra alrededor de un eje luzgravedad.

La morfometría dc un lago determina en gran medida los procesos biofísicos que tienen lugar en él (COLE, 1983; MARCALEF, 1983). En este sentido, el estudio morfométrico que se presenta tiene como objetivo el servir como elemento básico para posteriores estudios limnológicos.

Como antecedente a este trabajo existe una batimetría y propuesta de cartografía geológica del lago de Arreo elaborada por MARTÍNEZ-TORRES et al. (1992). El mapa batimétrico resultante. realizado con una metodología distinta, presenta notables diferencias con el que se presenta en el presente trabajo.

\section{MATERIAL Y MÉTODOS}

Las medidas de profundidad en la lámina de agua libre se realizaron con una ecosonda Furuno FE-4300. Se realizaron 14 transectos y numerosas medidas puntuales complementarias. En las zonas someras, donde la vegetación acuática emergente dificultaba la navegación, se realizaron medidas de forma manual con cinta métrica.

Como base cartográfica para el posicionamiento de puntos, medida de distancias, etc., se utilizó una fotografía aérea a escala 1:2000, ampliación del vuelo realizado el 21-4-92 por la Diputación Foral de Álava a escala 1:18000. Todos los datos fueron referenciados y tratados mediante un sistema de información geográfica (MAPINFO).

El estudio morfométrico sigue la metodología propuesta por HÅKANSON (1981).

\section{RESULTADOS Y DISCUSIÓN}

En el mapa batimétrico realizado por nosotros (Fig. 1) se observa que el lago está formado por una única cubeta asociada principalmente al cuadrante nororiental, donde se encuentran profundidades algo superiores a los $24 \mathrm{~m}$. Esta cubeta principal se corresponde con un tipo morfológico de dolina que se origina por el desarrollo de un criptokarst que provoca la génesis en superficie de una cubeta embudiforme (MONTES, 1996). 

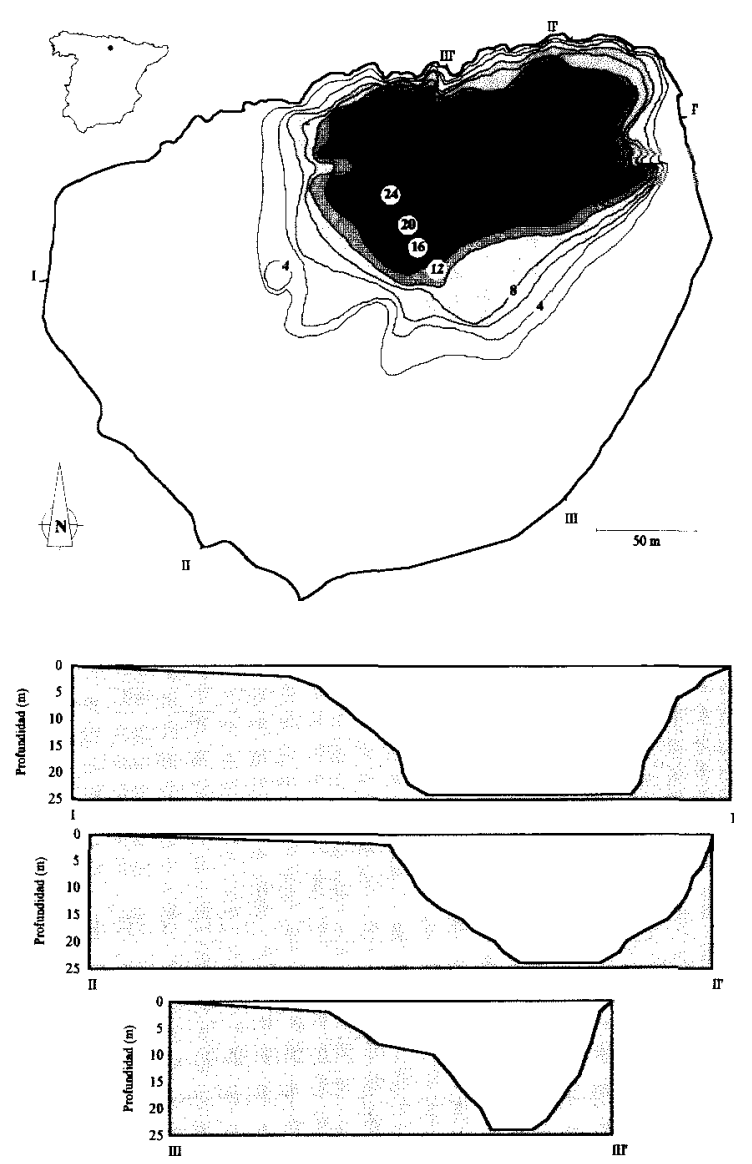

FIGURA 1. Mapa batimétrico y algunos perfiles del lago de Arreo. FIGURE 1. Bathymetric niap and several profiles of the lake of Arreo

Las máximas pendientes del lago se sitúan en la zona septentrional (próximas al 175\%), y parecen tener relación con una falla de dirección ENE-WSW (MARTÍNEZ-TORRES et al., 1992). En ese lado, el lago se encuentra limitado por un talud muy pronunciado que se corresponde con los materiales más duros (ofitas). Las menores pendientes (próximas al 0\%) se dan en la zona meridional y occidental, en donde dominan las arcillas.

Las principales diferencias respecto al mapa batimétrico previo realizado por MARTÍNEZ-TORRES et al. (1992) (Fig. 2) derivan de la subestima, por parte de éste, de las áreas someras. La evolución que ha tenido la banda de hidrófitos emergentes (Cladium mariscus y Phragmites australis, principalmente), la cual ha sido eliminada en parte por cortas e incendios provocados durante los últimos años, ha permitido en nuestro estudio delimitar más claramente cuáles son los límites de la lámina de agua en su zona litoral.

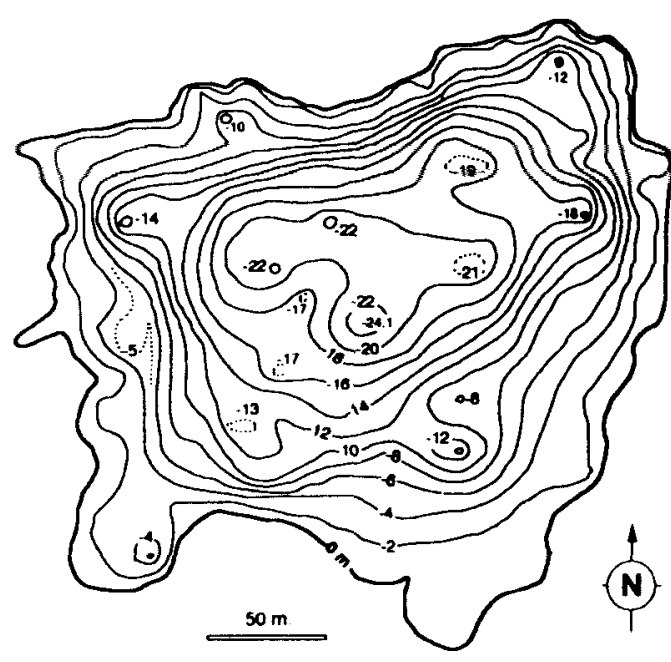

FIGURA 2. Mapa batimétrico previo del lago de Arreo (según MARTínEZTORRES et al., 1992)

FIGURE 2. Previous bathymetric map of the lake of Arreo (according to MARTÍNEZ-TORRES et al., 1992.

Las isolíneas de profundidad de nuestro mapa presentan, asímismo, una relación direccional mucho inás clara con la línea de falla que el mapa de MARTÍNEZ-TORRES et al. (op. cit.). Esta y otras diferencias de menor orden se derivan, sin duda, de la inetodología utilizada en el mapa previo, basado en un inuestreo de 60 localizaciones puntuales, sin ningún transecto continuo.

En la Tabla I se indican los valores de los parámetros morfométricos del lago según el mapa batimétrico presentado.

La relación entre la superficie del lago y la cuenca de drenaje es muy pequeña, lo cual, teóricamente, supondría un aporte limitado de nutrientes a la cubeta.

El desarrollo de costa es bajo, próximo a 1, como corresponde a cubetas formadas por disolución. La falla antes mencionada rompe la tendencia hacia la fonna circular que se adivina en el resto del lago. Esta forma circular, o inás bien subcircular, es otro aspecto que no se contemplaba bien en el mapa previo, de aspecto cuadrangular.

La profundidad relativa es muy grande $\left(\mathrm{D}_{\mathrm{r}}=8,57 \%\right)$, lo que es característico de lagos de pequeñas dimensiones. La profundidad media es mucho más grande que la mediana como corresponde a una forma global de la cubeta inuy convexa. Esta morfología se corresponde, asímisino, con el bajo desarrollo del volumen que presenta $\left(\mathrm{V}_{\mathrm{d}}=0,64\right)$.

La curva hipsográfica (Fig. 3) muestra que la forma del lago de Arreo es muy convexa en, aproximadamente, los 3/4 superiores de su área acumulada, pasando en el cuarto inferior 
'TABLA I. Parámetros morfométricos del lago de Arreo (Simbología según HÅKANSON, 1981)

TABLE I. Morphoriietrical parameters of the lake of Arreo (Symbols according to HAKANSON, 1981)

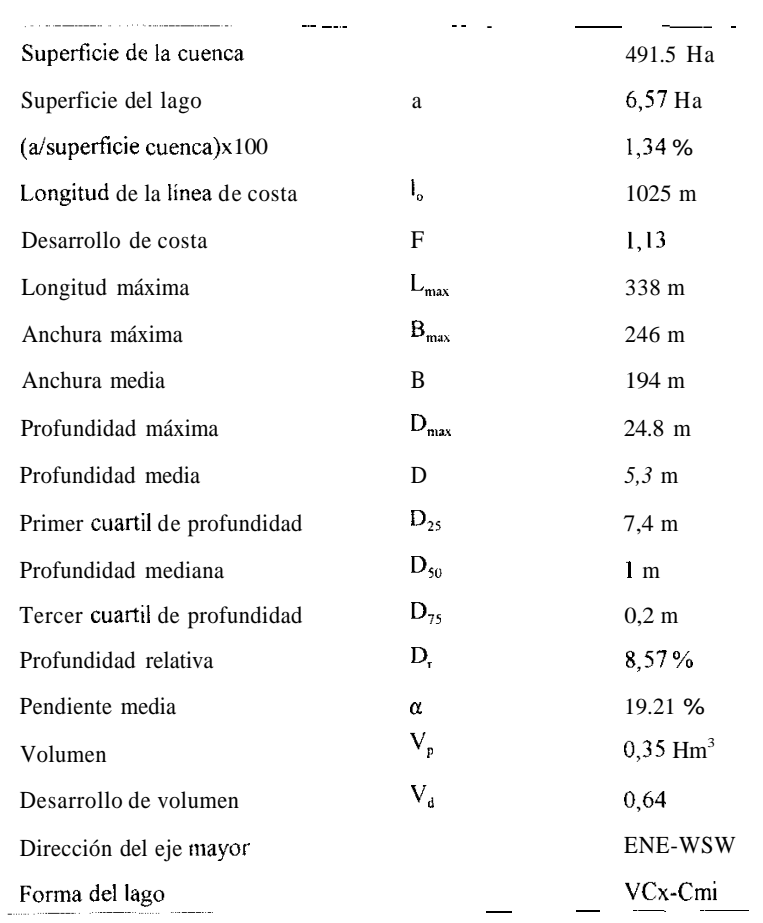

por las categorías de convexo, levemente convexo, linear e, incluso, en la zona más profunda, cóncavo. Utilizando la simbología de HÅKANSON (1981), y dado que se presentan algunos puntos de inflexión en la curva, el lago presenta una forma VCx-Cmi, que no resulta muy común dentro de la clasificación morfométrica lacustre. Se observa, así, un claro predorninio de las zonas poco profundas del lago, situándose las inayores profundidades en una superficie pequeña hablando en términos relativos.

Teniendo en cuenta el funcionainiento térinico del lago (GONZALEZ et al., en prep.), éste estaría sometido a una dinámica de tipo palustre (mezcla permanente) en, al menos, 2/3 de su superficie, quedando relegada a tina dinámica estrictamente lacustre (estratificación térmica estacional) en un área sensiblemente menor.

El lago de Arreo debe considerarse, de esta forma, como un sistema acuático forinado por dos subsistemas, lacustre y palustre, íntimamente relacionados. El subsistema palustre, tal como lo hemos entendido hasta el momento, englobado dentro de la lámina de agua hasta una profundidad de $3-4 \mathrm{~m}$, con tina morfometría de pendientes suaves y con un sustrato colonizado por macrófitos acuáticos sumergidos y helófitos, tiene en Arreo una continuidad, desde el punto de vista ecológico, en la

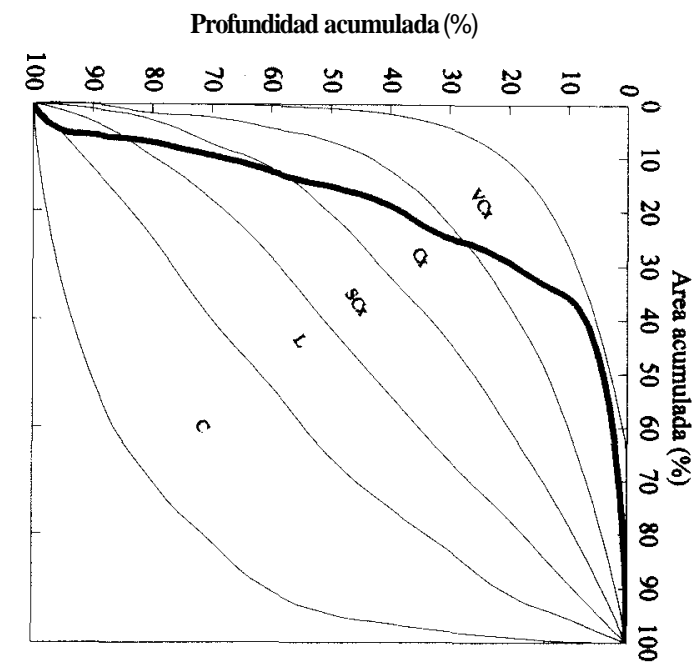

Profundidad acumulada (\%)

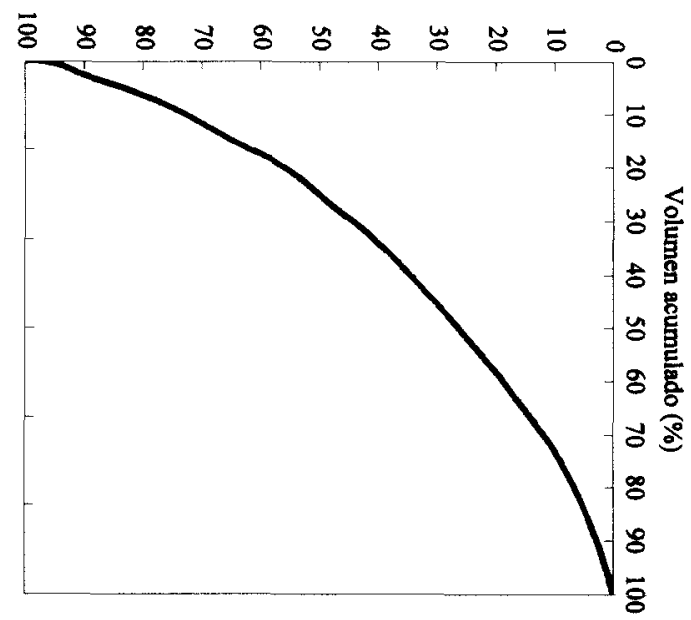

FIGURA 3. Curvas hipsográfica y de volumen relativas del lago de Arreo (se indican los símbolos y límites de clases del sistema de clasificación morfológica lacustre según HÅKANSON, 1981)

FIGURE 3. Relative hyposographic and volume curves for the lake of Arreo (symbols and class limits for the classification system of lake forms accoding to HAKANSON (1981) are indicated.

interfase tierra-agua hasta un límite exterior difícil de delimitar físicamente pero que, en el lago, se manifiesta por un gran desarrollo de la comunidad de helófitos. Este conjunto, que podría tomar la denominación de subsistema litoral, controlaría en gran medida el metabolismo global del lago en su conjunto al controlar la disponibilidad de nutrientes (WETZEL, 1990).

Es de notar que el funcionamiento térmico del lago presenta una gran estabilidad en la estratificación, aspecto éste favorecido por las características morfométricas del lago. Parámetros como la grandísima profundidad relativa que presenta son usados para describir esta propiedad 
(HÅKANSON, 1981), así como el desarrollo de máximos de oxígeno metalimnético (EBERLY, 1964), que en el lago de Arreo son muy pronunciados, pudiendo alcanzar picos de oxígeno con una sobresaturación cercana al $200 \%$.

El lago de Arreo se ve sometido los últimos años a la extracción de agua en época estival para su utilización en el riego de cultivos circundantes, disminuyendo el nivel del lago unos $2 \mathrm{~m}$. Analizando la evolución de la superficie y volumen acumulados del lago (Fig. 3, Tabla II) vemos como este impacto afecta a más de la mitad dc la superficie del mismo, extrayéndose alrededor de 1/4 de su volumen.

TABLA II. Superficie y volumen acumulados del lago de Arreo. TABLE II Cummulative area and volume of the lake of Arreo.

\begin{tabular}{ccc}
$\begin{array}{c}\text { Profundidad } \\
(\mathrm{m})\end{array}$ & $\begin{array}{c}\text { Superficie acumulada } \\
(\mathrm{Ha})\end{array}$ & $\begin{array}{c}\text { Volumen acumulado } \\
\left(\mathrm{Hm}^{3}\right)\end{array}$ \\
\hline 0 & 6,57 & 0,349 \\
2 & 2,47 & 0,269 \\
4 & 2,08 & 0,224 \\
6 & 1,79 & 0,185 \\
8 & 1,58 & 0,150 \\
10 & 1,27 & 0,120 \\
12 & 1,08 & 0,094 \\
14 & 0,93 & 0,072 \\
16 & 0,75 & 0,052 \\
18 & 0,61 & 0,035 \\
20 & 0,48 & 0,021 \\
22 & 0,36 & 0,009 \\
24 & 0,23 & 0,000 \\
24,8 & 0 & 0,000 \\
\hline
\end{tabular}

Desde el punto de vista morfométrico, la alteración más evidente que se manifiesta en el lago deriva del derrumbe del talud asociado a la falla del lado norte, que afecta a la zona más profunda del lago. Este hecho es consecuencia, en gran parte, de la periódica fluctuación del nivel de la lámina de agua, que altera la cimentación del talud. Es de destacar que este fenómeno se ha dado anteriormente de forma natural, habiéndose encontrado alguna capa de sedimentos derivados, sin duda, de derrumbes en el análisis de cilindros de sedimentos extraídos de la cubeta más profunda. No obstante, es evidente que la frecuencia de dichos procesos se ha incrementado con las actuaciones antrópicas descritas. El mantenimiento de la morfometría actual del lago, por no hablar de los procesos ecológicos del subsistema litoral, requiere, por lo tanto, el cese de dichas actuaciones.

\section{BIBLIOGRAFÍA}

COLE, G.A. 1983. Textbook of limnology. The C.V. Mosby Company. St. Louis. 3rd edition.

EBERLY, W.R. 1964. Further studies on the metalimnetic oxygen maximum, with special reference to its occurrence throughout the world. Investigations of Indiana Lake Streams, vol. 6 .

HÅKANSON, L. 1981. A manual of lake morphometry. Springer-Verlag. Berlín, Heidelberg, New York. 78 págs.

MARGALEF, R. 1983. Limnología. Ed. Omega. Barcelona. MARTÍNEZ-TORRES, L.M.; GONZÁLEZ-TAPIA, J.R. \& R. RAMÓM-LLUCH. 1992. Batimetría y propuesta de cartografía geológica del lago de Arreo (Diapiro de Salinas de Añana, Álava). Eusko Ikaskuntza. Cuadernos de Sección. Historia, 20: 123-134.

MONTES, C. (coord.). 1996. Clasificación genético-funcional de los lagos y humedales de España. En: Proyectos de apoyo técnico a los planes hidrológicos de cuenca en aspectos relacionados con zonas húmedas. Dirección. General de Obras Hidráulicas. MOPTMA. INIMA.

RÍOS, J.M. 1963. Materiales salinos del suelo español. Instituto Geológico y Minero de España. Memoria 64. 166 págs.

WETZEL, R.G. 1990. Land-water interfaces. Metabolic and limnological regulators. Ver. Internat. Verein Limnol., 24: 6-24. 